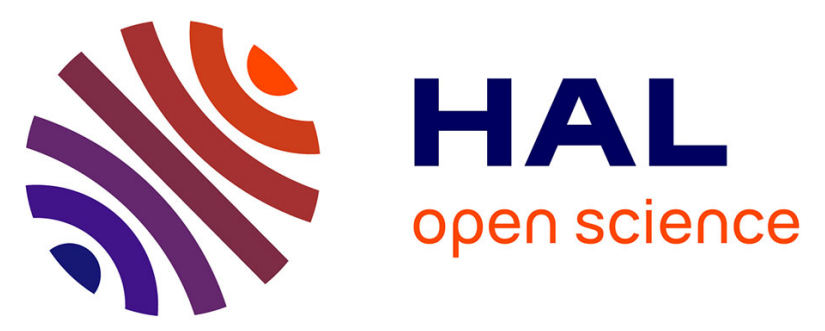

\title{
Towards robust viscoelastic-plastic-damage material model with different hardenings / softenings capable of representing salient phenomena in seismic loading applications
}

\author{
Pierre Jehel, Luc Davenne, Adnan Ibrahimbegovic, Pierre Léger
}

\section{To cite this version:}

Pierre Jehel, Luc Davenne, Adnan Ibrahimbegovic, Pierre Léger. Towards robust viscoelastic-plasticdamage material model with different hardenings / softenings capable of representing salient phenomena in seismic loading applications. Computers and Concrete, an International Journal, 2010, 7 (4), pp.365-386. hal-00469687v2

\section{HAL Id: hal-00469687 \\ https://hal.science/hal-00469687v2}

Submitted on 3 Apr 2010

HAL is a multi-disciplinary open access archive for the deposit and dissemination of scientific research documents, whether they are published or not. The documents may come from teaching and research institutions in France or abroad, or from public or private research centers.
L'archive ouverte pluridisciplinaire HAL, est destinée au dépôt et à la diffusion de documents scientifiques de niveau recherche, publiés ou non, émanant des établissements d'enseignement et de recherche français ou étrangers, des laboratoires publics ou privés. 


\title{
Towards robust viscoelastic-plastic-damage material model with different hardenings / softenings capable of representing salient phenomena in seismic loading applications
}

\author{
Jehel $P^{1,2}$, Davenne $L^{1}$, Ibrahimbegovic $A^{1}$, Léger $P^{2}$ \\ 1. LMT-Cachan (ENS Cachan/CNRS/UPMC/PRES UniverSud Paris) \\ 61 av. du Président Wilson, 94230 Cachan, France
}

2. Department of Civil Engineering, École Polytechnique de Montréal University of Montreal Campus, P.O. Box 6079, Station CV, Montreal, PQ, Canada H3C 3A7

Corresponding author:

Name: Adnan Ibrahimbegovic

Phone: +33 (0) 147402234

Fax: +33 (0) 147402240

Email: ai@lmt.ens-cachan.fr

\begin{abstract}
This paper presents the physical formulation of a 1D material model suitable for seismic applications. It is written within the framework of thermodynamics with internal variables that is, especially, very efficient for the phenomenological representation of material behaviors at macroscale: those of the representative elementary volume. The model can reproduce the main characteristics observed for concrete, that is nonsymetric loading rate-dependent (viscoelasticity) behavior with appearance of permanent deformations and local hysteresis (continuum plasticity), stiffness degradation (continuum damage), cracking due to displacement localization (discrete plasticity or damage). The parameters have a clear physical meaning and can thus be easily identified. Although this point is not detailed in the paper, this material model is developed to be implemented in a finite element computer program. Therefore, for the benefit of the robustness of the numerical implementation, (i) linear state equations (no local iteration required) are defined whenever possible and (ii) the conditions in which the presented model can enter the generalized standard materials class - whose elements benefit from good global and local stability properties - are clearly established. To illustrate the capabilities of this model - among them for Earthquake Engineering applications - results of some numerical applications are presented.
\end{abstract}

Keywords: Thermodynamics with internal variables; Phenomenological approach; Continuum-discrete plasticity and damage models; Generalized standard material; Earthquake Engineering. 


\section{Introduction}

The development of numerical computing facilities within the last decades has made it possible to carry out numerical large scale dynamic nonlinear analyses that take into account the nonlinear behavior of the materials, such as steel and concrete in reinforced concrete structures $[1,6,23,25]$. Multi-scale-like stategies for defining frame section behavior laws from information collected at the material level also emerged, e.g. for static ultimate load design [30]. The implementation of robust refined material models in computer programs has thus become a key issue in structural engineering. Indeed, the prediction of the timehistory of some material properties provides useful information to predict the global structural seismic response: strength and stiffness degradations as well as residual deformation in the materials help for drawing conclusions about the residual capacity of the structure in post-earthquake conditions. In Earthquake Engineering, the physical modeling of damping is not an easy task because many dissipative phenomena occur at several levels. Refined material models are required for a reliable computation of the amount of material energy dissiped in seismic loading. Other dissipation sources at the level of the bounding between steel and concrete in RC structural elements have already been introduced in numerical static analyses $[8,17]$ and one can hope that they will be adapted to seismic analyses within the next few years. Since many computations are required for complete seismic analyses (need to consider several input motions) or in automatic parameters identification procedures [19], the robustness of the material models should be considered with care.

This paper deals with modeling the behavior of concrete until collapse in dynamic loading applications so that it is robust once implemented in a computer program. The authors already presented a robust elasto-plastic-damage model and its implementation in finite element procedures [18]. In addition here, in the physical formulation: (i) viscosity is added by appealing to the so-called Kelvin-Voigt viscoelastic model; (ii) the modeling of the softening part, which is introduced in the formulation by a discrete plastic or damage model that requires the definition of an enhanced strain field, is presented in detail and (iii) a clear distinction between tensile and compressive constitutive models is made. On the numerical side, the conditions under which the model can enter the generalized standard materials class [11], whose elements benefit from good robustness properties, are identified. This latter point, along with the model formulation that allows solving the local problem (the integration point level) without any iteration, except in tension, in a numerical nonlinear structural analysis [18] justifies the use of the words "towards robust" in this paper title.

This material model is formulated in the framework provided by thermodynamics with internal variables (TIV), which is very suitable for developing phenomenological material constitutive models with good numerical properties [24]. A set of internal variables is chosen to describe nonlinear phenomena that occur in the material. Our approach is phenomenological because the nonlinear phenomena we aim at modeling for concrete are those observed on the strain-stress experimental macroscopic response of a concrete representative elementary volume (REV). However, internal variables often are connected to microscopic phenomena that have macroscopic effects such as in steel materials where microscopic dislocations motion lead to a macroscopic strain hardening behavior. More accurate representations of concrete could be provided for numerical 
simulations by, for instance, taking into account the heterogeneous nature of concrete [12]. The macroscopic phenomenological approach is however an efficient way to carry out large-scale structural numerical simulations with a rather refined material description.

The introduction of viscosity in a material model can be motivated by several purposes. (i) Viscosity in concrete can be associated to its loading ratedependent macroscopic behavior that results from several nano- and microscopic phenomena such as complex interaction between moisture and micro-structural solid skeleton, micro-cracking process, Stefan effect, micro-inertia of the material surronding the crack tip [28]. (ii) Viscosity can also be associated to creep phenomena in highly dissipative materials such as asphalt concrete [27]. (iii) The introduction of viscosity leads to the explicit appearance of a time-step in the governing equations of the discretized problem. The possibility to determine a critical time-step then provides a tool to reguralize numerical problems that can be ill-posed in the presence of materials that exhibit strain softening behaviors [26]. (iv) Viscosity can also be used to introduce a source of energy dissipation at the material level: it can lump the energy dissipation that physically comes from unknown or not (accurately) modeled physical nonlinear phenomena in the materials; this is what the authors have in mind when developing this concrete constitutive model.

The aim of this paper is to present the theoretical formulation of a refined 1D cyclic constitutive concrete model that has been developed in a framework leading to a robust implementation into finite element numerical procedures. We first present in section 2 the theoretical formulation of the constitutive concrete model; in section 3, the conditions in which the model can enter the generalized standard material class are examined for the benefit of robustness; in section 4 , the mechanical problem of a concrete $1 \mathrm{D}$ structure in dynamic loading is formulated in a complementary variational form and the Euler-Lagrange equations are derived; before to conclude, section 5 is dedicated to the presentation of some numerical examples that illustrate some capabilities of the constitutive concrete model implemented in a finite element program.

\section{Constitutive concrete model}

\subsection{Thermodynamics with internal variables}

To represent the salient phenomena observed in the experimental cyclic response of a concrete REV (Fig. 1), a set $\boldsymbol{\alpha}$ of internal variables is chosen. The phenomena we aim at modeling are: (i) loading-rate dependent behavior, (ii) strain hardening, (iii) strain softening, (iv) appearance of residual deformation, (v) stiffness and strength degradations, (vi) hysteresis loops. We consider that the phenomena (ii), (iv) and (vi) only occur in compression, that strain softening in compression is due to the localization of permanent deformation whereas strain softening in tension is due to the localization of deformation that completely disappear after unloading. Internal variables are associated to each of these phenomenologically identified mechanisms.

The set of internal variables we choose is defined in Tab. 1. $\boldsymbol{\bullet}$ and $\overline{\boldsymbol{\bullet}}$ refer to continuum and discrete quantities. Concerning the continuum internal 

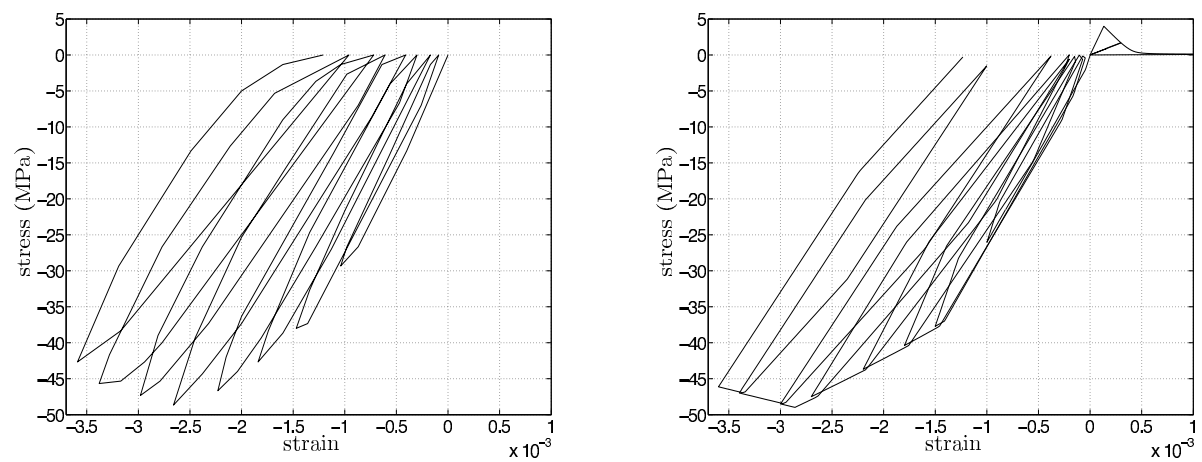

Fig. 1: [left] Experimental (adapted from [31]) and [right] numerical (by using the proposed constitutive concrete model) cyclic behavior of concrete in quasi-static loading.

variables: $\bar{\epsilon}^{v}$ and $\bar{\epsilon}^{p}$ are the viscous and plastic deformations, $\bar{\xi}^{p}$ and $\bar{\lambda}^{p}$ are the strain-like variables that represent isotropic and kinematic plastic strain hardenings, $\bar{D}$ represents stiffness degradation and $\bar{\xi}^{d}$ isotropic damage strain hardening. Then, concerning the discrete internal variables, that is in the section where displacement localizes, $\overline{\bar{u}}^{p}$ represents plastic deformation, $\overline{\bar{\xi}}^{p}$ plastic strain softening in compression, $\overline{\bar{D}}$ the stiffness degradation and $\overline{\bar{\xi}}^{d}$ damage strain softening in tension.

\begin{tabular}{|l|c|ccccccc|cc|}
\hline & $\mathrm{t} / \mathrm{c}$ & \multicolumn{10}{|c|}{ compression } & \multicolumn{2}{|c|}{ tension } \\
\hline & & & & & & & & \\
$\boldsymbol{\alpha}$ & $\bar{\epsilon}^{v}$ & $\bar{\epsilon}^{p}$ & $\bar{\xi}^{p}$ & $\bar{\lambda}^{p}$ & $\bar{D}$ & $\bar{\xi}^{d}$ & $\overline{\bar{u}}^{p}$ & $\overline{\bar{\xi}^{p}}$ & $\overline{\bar{D}}$ & $\overline{\bar{\xi}}^{d}$ \\
$\mathcal{A}$ & $\sigma^{v}$ & $\sigma$ & $\bar{q}^{p}$ & $\bar{\kappa}^{p}$ & $\frac{\sigma^{2}}{2}$ & $\bar{q}^{d}$ & $\mathrm{t}$ & $\overline{\bar{q}}^{p}$ & $\frac{\mathrm{t}}{2}^{2}$ & $\overline{\bar{q}}^{d}$ \\
$\mathrm{PC}$ & $E, \eta$ & $\bar{E}$ & $\bar{K}^{p}$ & $\bar{H}^{p}$ & $\bar{E}^{-1}$ & $\bar{K}^{d}$ & $\overline{\bar{E}}$ & $\overline{\bar{K}}^{p}$ & $\overline{\bar{E}}^{-1}$ & $\sigma_{\infty}, a$ \\
\hline
\end{tabular}

Tab. 1: Set of internal variables $\boldsymbol{\alpha}$, corresponding affinities $\mathcal{A}$ and phenomenological coefficients PCs (parameters of the model).

A noteworthy advantage of this material model is that all its parameters have a clear meaning and can therefore be easily identified according to experimental curves. The complete procedure to identify a concrete law is presented in Section 5.1: the viscous parameter $\eta$ can be seen as a material property and identified from free vibration tests; the tensile softening curve coefficient $a$ is related to the fracture energy $G_{F}$, a fracture mechanics concept [13] that quantifies the total amount of energy that has to be furnished in tension to a concrete section between the time when displacement begins to localize and the time when the section is completely broken; all the other parameters can be identified from stress-strain curves of concrete specimen in quasi-static cyclic loading. 


\subsection{Governing equations of the constitutive model}

\subsubsection{Basic ingredients}

We first define the three basic ingredients for developing a constitutive model in the framework of the $T I V$ :

1. Split of the total strain into a viscoelastic, a plastic, a damage and another discrete part due to the likely localization of the displacement. $\mathcal{A}$-space is devided into two parts: the terms involved in tension $\sigma \geq 0$ are mentioned by the sign $\bullet+$ and those involved in compression $\sigma<0$ by the sign $\bullet^{-}$ (note that for a 1D case, tension and compression have a clear meaning):

$$
\begin{aligned}
u(x, t) & =\bar{u}(x, t)+\overline{\bar{u}}(t) \mathcal{H}_{\bar{x}}(x) \\
\epsilon(x, t) & =\frac{\partial u}{\partial x}=\bar{\epsilon}^{v}+\bar{\epsilon}^{p}+\left(\overline{\bar{u}}^{\diamond}+\overline{\bar{u}}^{p}\right) \delta_{\bar{x}}
\end{aligned}
$$

where $\bar{\epsilon}=\frac{\partial \bar{u}}{\partial x}=\bar{\epsilon}^{v}+\bar{\epsilon}^{p}$ is the continuum part of the strain and $\overline{\bar{u}} \delta_{\bar{x}}=$ $\left(\overline{\bar{u}}^{\diamond}+\overline{\bar{u}}^{p}\right) \delta_{\bar{x}}-$ with $\overline{\bar{u}}^{\diamond}=\overline{\bar{u}}^{d}$ in tension and $\overline{\bar{u}}^{\diamond}=\overline{\bar{u}}^{e}$ in compression - its discrete part introduced by the Dirac's function $\delta_{\bar{x}}$ that is the derivative of the Heaviside's function $\mathcal{H}_{\bar{x}}$ that introduces the displacement jump $\overline{\bar{u}}$ in the description of the displacement. $\bar{\epsilon}^{v}$ corresponds to the deformation in a Kelvin-Voigt rheological model: a spring - elastic in tension $\left(\bar{\epsilon}^{v}=\bar{\epsilon}^{e}\right)$ and damaging in compression $\left(\bar{\epsilon}^{v}=\bar{\epsilon}^{d}\right)$ - and a dashpot in parallel. For simplicity, we assume here that the displacement jumps only take place in one section; extension to several localization sections is straightforward.

2. Helmholtz free energy functional:

$$
\begin{aligned}
\psi^{+}(u, \boldsymbol{\alpha})= & \bar{\psi}^{e}\left(\bar{u}^{+}\right)+\left(\overline{\bar{\psi}}^{d}\left(\overline{\bar{u}}^{+}, \overline{\bar{D}}\right)+\overline{\bar{\Xi}}^{d}\left(\overline{\bar{\xi}}^{d}\right)\right) \delta_{\bar{x}} \\
\psi^{-}(u, \boldsymbol{\alpha})= & \bar{\psi}^{d}\left(\bar{u}^{-}, \bar{\epsilon}^{p}, \bar{D}\right)+\bar{\Xi}^{p}\left(\bar{\xi}^{p}\right)+\bar{\Lambda}^{p}\left(\bar{\lambda}^{p}\right)+\bar{\Xi}^{d}\left(\bar{\xi}^{d}\right)+ \\
& \left(\overline{\bar{\psi}}^{e}\left(\overline{\bar{u}}^{-}, \overline{\bar{u}}^{p}\right)+\overline{\bar{\Xi}}^{p}\left(\overline{\bar{\xi}}^{p}\right)\right) \delta_{\bar{x}}
\end{aligned}
$$

3. The nonlinear plasticity and damage mechanisms are activated for a positive value of the corresponding plasticity and damage criteria:

- For $\sigma \geq \mathbf{0}$ (tension), the dissipative mechanisms that can be involved in the evolution process are activated according to the following criteria:

$$
\begin{aligned}
& \bar{\phi}^{v,+}(\sigma)=\sigma^{v}=\sigma-\sigma^{e} \leq 0 \\
& \overline{\bar{\phi}}^{d,+}\left(\mathrm{t}, \overline{\bar{q}}^{d}\right)=\mathrm{t}-\left(\sigma_{u}^{t}-\overline{\bar{q}}^{d}\right) \leq 0
\end{aligned}
$$

where $\mathrm{t}$ is the stress at the discontinuity and $\sigma_{u}^{t}$ is the ultimate stress in tension.

- For $\sigma<\mathbf{0}$ (compression), the criteria functions are:

$$
\begin{aligned}
& \bar{\phi}^{v,-}(\sigma)=-\sigma^{v}=-\left(\sigma-\sigma^{d}\right) \leq 0 \\
& \bar{\phi}^{p,-}\left(\sigma, \bar{q}^{p}, \bar{\kappa}^{p}\right)=\left|\sigma+\bar{\kappa}^{p}\right|-\left(\sigma_{y}-\bar{q}^{p}\right) \leq 0 \\
& \bar{\phi}^{d,-}\left(\sigma, \bar{q}^{d}\right)=-\sigma-\left(\sigma_{f}-\bar{q}^{d}\right) \leq 0 \\
& \overline{\bar{\phi}}^{p,-}\left(\mathrm{t}, \overline{\bar{q}}^{p}\right)=-\mathrm{t}-\left(\sigma_{u}^{c}-\overline{\bar{q}}^{p}\right) \leq 0
\end{aligned}
$$


where $\sigma_{y}, \sigma_{f}$ and $\sigma_{u}^{c}$ are yield, fracture and ultimate stresses in compression.

\subsubsection{State equations of the system}

We can now give the expression of the energy dissipated by this viscoelasticplastic-damage model with different hardenings / softenings within a time unit. This can be computed as the difference between the total amount of energy imparted to the system and the amount of energy stored by the system (internal potential energy) during this time unit:

\section{In tension:}

$$
\begin{aligned}
\dot{\mathcal{D}}^{+} & =\left(\sigma \dot{\bar{\epsilon}}^{+}+\mathrm{t} \dot{\overline{\bar{u}}}^{+} \delta_{\bar{x}}\right)-\dot{\psi}^{+}\left(\bar{u}^{+}, \overline{\bar{u}}^{+}, \overline{\bar{D}}, \overline{\bar{\xi}}^{d}\right) \\
& =\left(\sigma^{e}+\sigma^{v}\right) \dot{\bar{\epsilon}}^{+}+\mathrm{t} \dot{\overline{\bar{u}}}^{+} \delta_{\bar{x}}-\frac{\partial \bar{\psi}^{e}}{\partial \bar{\epsilon}^{+}} \dot{\bar{\epsilon}}^{+}-\left(\frac{\partial \overline{\bar{\psi}}^{d}}{\partial \overline{\bar{u}}^{+}} \dot{\overline{\bar{u}}}^{+}+\frac{\partial \overline{\bar{\psi}} d}{\partial \overline{\bar{D}}} \dot{\overline{\bar{D}}}+\frac{\partial \overline{\bar{\Xi}}^{d}}{\partial \overline{\bar{\xi}}^{d}}\right) \delta_{\overline{\bar{x}}}
\end{aligned}
$$

We also have $\dot{\bar{\epsilon}}^{+}=\dot{\bar{\epsilon}}^{e}=\dot{\bar{\epsilon}}^{v}$ and $\dot{\overline{\bar{u}}}^{+}=\dot{\overline{\bar{u}}}^{d}$ because $\overline{\bar{u}}^{p}$ cannot evolve in tension according to Eqs. (3) and (4). Thus:

$$
\dot{\mathcal{D}}^{+}=\left(\sigma^{e}-\frac{\partial \bar{\psi}^{e}}{\partial \bar{\epsilon}^{e}}\right) \dot{\bar{\epsilon}}^{e}+\sigma^{v,+} \dot{\bar{\epsilon}}^{v,+}+\left(\mathrm{t}-\frac{\partial \overline{\bar{\psi}}^{d}}{\partial \overline{\bar{u}}^{d}}\right) \dot{\overline{\bar{u}}}^{d} \delta_{\bar{x}}-\frac{\partial \overline{\bar{\psi}} d}{\partial \overline{\bar{D}}} \dot{\overline{\bar{D}}} \delta_{\bar{x}}-\frac{\partial \overline{\overline{\bar{\Xi}}} d}{\partial \overline{\bar{\xi}} d} \dot{\bar{\xi}}^{d} \delta_{\bar{x}}
$$

\section{In compression:}

$$
\begin{aligned}
\dot{\mathcal{D}}^{-}= & \left(\sigma \dot{\bar{\epsilon}}^{-}+\mathrm{t} \dot{\overline{\bar{u}}}^{-} \delta_{\bar{x}}\right)-\dot{\psi}^{-}\left(\bar{u}^{-}, \overline{\bar{u}}^{-}, \bar{\epsilon}^{p}, \bar{\xi}^{p}, \bar{\lambda}^{p}, \bar{D}, \bar{\xi}^{d}, \overline{\bar{u}}^{p}, \overline{\bar{\xi}}^{p}\right) \\
= & \left(\sigma^{d}+\sigma^{v}\right) \dot{\bar{\epsilon}}^{-}+\mathrm{t} \dot{\bar{u}}^{-} \delta_{\bar{x}}-\left(\frac{\partial \bar{\psi}^{d}}{\partial\left(\bar{\epsilon}^{-}-\bar{\epsilon}^{p}\right)}\left(\dot{\bar{\epsilon}}^{-}-\dot{\bar{\epsilon}}^{p}\right)+\frac{\partial \bar{\psi}^{d}}{\partial \overline{\bar{D}}} \dot{\bar{D}}+\frac{\partial \overline{\bar{\Xi}}^{p}}{\partial \overline{\bar{\xi}}^{p}} \dot{\bar{\xi}}^{p}\right. \\
& \left.+\frac{\partial \overline{\bar{\Lambda}}^{p}}{\partial \overline{\bar{\lambda}}^{p}} \dot{\bar{\lambda}}^{p}+\frac{\partial \bar{\Xi}^{d}}{\partial \overline{\bar{\xi}}^{d}} \dot{\bar{\xi}}^{d}\right)-\left(\frac{\partial \overline{\bar{\psi}}^{e}}{\partial\left(\overline{\bar{u}}^{-}-\overline{\bar{u}}^{p}\right)}\left(\dot{\overline{\bar{u}}}^{-}-\dot{\overline{\bar{u}}}^{p}\right)+\frac{\partial \overline{\bar{\Xi}}^{p}}{\partial \overline{\bar{\xi}}^{p}} \dot{\bar{\xi}}^{p}\right) \delta_{\bar{x}}
\end{aligned}
$$

We also have $\dot{\bar{\epsilon}}^{-}=\dot{\bar{\epsilon}}^{v}+\dot{\bar{\epsilon}}^{p}$ with $\dot{\bar{\epsilon}}^{v}=\dot{\bar{\epsilon}}^{d}$ and $\dot{\overline{\bar{u}}}^{-}=\dot{\overline{\bar{u}}}^{e}+\dot{\overline{\bar{u}}}^{p}$. Thus:

$$
\begin{aligned}
\dot{\mathcal{D}}^{-}= & \left(\sigma^{d}-\frac{\partial \bar{\psi}^{d}}{\partial\left(\bar{\epsilon}^{-}-\bar{\epsilon}^{p}\right)}\right) \dot{\bar{\epsilon}}^{d}+\sigma^{v} \dot{\bar{\epsilon}}^{v,-}+\sigma \dot{\bar{\epsilon}}^{p}-\frac{\partial \bar{\psi}^{d}}{\partial \bar{D}} \dot{\bar{D}}-\frac{\partial \bar{\Xi}^{p}}{\partial \bar{\xi}^{p}} \dot{\bar{\xi}}^{p}-\frac{\partial \bar{\Lambda}^{p}}{\partial \bar{\lambda}^{p}} \dot{\bar{\lambda}}^{p} \\
& -\frac{\partial \bar{\Xi}^{d}}{\partial \overline{\bar{\xi}}^{d}} \dot{\bar{\xi}}^{d}+\left(\mathrm{t}-\frac{\partial \overline{\bar{\psi}}^{e}}{\partial\left(\overline{\bar{u}}--\overline{\bar{u}}^{p}\right)}\right) \dot{\overline{\bar{u}}}^{e} \delta_{\bar{x}}+\mathrm{t} \dot{\bar{u}}^{p} \delta_{\bar{x}}-\frac{\partial \overline{\bar{\Xi}}^{p}}{\partial \overline{\bar{\xi}}^{p}} \dot{\bar{\xi}}^{p} \delta_{\bar{x}}
\end{aligned}
$$

Note that $\bar{\epsilon}^{e}, \overline{\bar{u}}^{d}, \bar{\epsilon}^{d}, \overline{\bar{u}}^{e}$ are not internal variables. Moreover, when there is no evolution of any internal variable, the dissipation is null. Therefore, according to Eqs. (5) and (6), we have the following state equations:

$$
\sigma^{e}=\frac{\partial \bar{\psi}^{e}}{\partial \bar{\epsilon}^{e}} \quad, \quad \sigma^{d}=\frac{\partial \bar{\psi}^{d}}{\partial \bar{\epsilon}^{d}} \quad, \quad \mathrm{t}^{+}=\frac{\partial \overline{\bar{\psi}}^{d}}{\partial \overline{\bar{u}}^{d}} \quad, \quad \mathrm{t}^{-}=\frac{\partial \overline{\bar{\psi}}^{e}}{\partial \overline{\bar{u}}^{e}}
$$

To recover, for the thermodynamic forces, expressions that will then lead to an accurate reproduction of the experimentally observed behavior of a concrete 
REV, we define the different terms in the expression of the Helmholtz free energy as follows:

$$
\begin{aligned}
\bar{\psi}^{e}=\frac{1}{2} \bar{\epsilon}^{e} \bar{E} \bar{\epsilon}^{e} & \bar{\psi}^{d}=\frac{1}{2}\left(\bar{\epsilon}^{-}-\bar{\epsilon}^{p}\right) \bar{D}^{-1}\left(\bar{\epsilon}^{-}-\bar{\epsilon}^{p}\right) \\
\overline{\bar{\psi}}^{d}=\frac{1}{2} \overline{\bar{u}}^{d} \overline{\bar{D}}^{-1} \overline{\bar{u}}^{d} & \bar{\Xi}^{p}=\frac{1}{2} \bar{\xi}^{p} \bar{K}^{p} \bar{\xi}^{p} \\
\overline{\bar{\Xi}}^{d}=-\left(\sigma_{u}^{t}-\sigma_{\infty}\right)\left(\overline{\bar{\xi}}^{d}+\frac{1}{a} e^{-a \bar{\xi}^{d}}\right) & \bar{\Lambda}^{p}=\bar{\lambda}^{p} \bar{H}^{p} \bar{\lambda}^{p} \\
\bar{\Xi}^{d} & =\frac{1}{2} \bar{\xi}^{d} \bar{K}^{d} \bar{\xi}^{d} \\
\overline{\bar{\psi}}^{e} & =\left(\overline{\bar{u}}^{-}-\overline{\bar{u}}^{p}\right) \overline{\bar{E}}\left(\overline{\bar{u}}^{-}-\overline{\bar{u}}^{p}\right) \\
& \overline{\bar{\Xi}^{p}}=\frac{1}{2} \overline{\bar{\xi}}^{p} \overline{\bar{K}}^{p} \overline{\bar{\xi}}^{p}
\end{aligned}
$$

We define the state equations of the internal variables from the Helmholtz free energy functional that plays the role of a thermodynamic potential:

$$
\mathcal{A}_{i}=-\frac{\partial \psi}{\partial \alpha_{i}}
$$

Finally, the state equations of the system, that is the equations that characterize the state of a concrete material point at a given instant in $\mathcal{A}$-space, are given in Tab. 2.

\begin{tabular}{ll}
\hline Tension & Compression \\
\hline$\sigma^{e}=\bar{E} \bar{\epsilon}^{e}$ & $\sigma^{d}=\bar{D} \bar{\epsilon}^{d}$ \\
$\mathrm{t}=\overline{\bar{D}}^{-1} \overline{\bar{u}}^{d}$ & $\mathrm{t}=\overline{\bar{E}} \overline{\bar{u}} e^{e}$ \\
$\overline{\bar{q}}^{d}=-\frac{\partial \overline{\bar{\Xi}}^{d}}{\partial \bar{\xi}^{d}}=\left(\sigma_{u}^{t}-\sigma_{\infty}\right)\left(1-e^{-a \bar{\xi}^{d}}\right)$ & $\bar{q}^{p}=-\frac{\partial \bar{\Xi}^{p}}{\partial \bar{\xi}^{p}}=-\bar{K}^{p} \bar{\xi}^{p}$ \\
$\frac{1}{2} \mathrm{t}^{2}=-\frac{\partial \bar{\psi}^{d}}{\partial \bar{D}}$ & $\bar{\kappa}^{p}=-\frac{\partial \bar{\Lambda}^{p}}{\partial \lambda^{p}}=-\bar{H}^{p} \bar{\lambda}^{p}$ \\
& $\bar{q}^{d}=-\frac{\partial \bar{\Xi}^{d}}{\partial \bar{\xi}^{d}}=-\bar{K}^{d} \bar{\xi}^{d}$ \\
& $\frac{1}{2} \sigma^{2}=-\frac{\partial \bar{\psi}^{d}}{\partial \bar{p}^{D}}$ \\
& $\bar{q}^{p}=-\frac{\partial \overline{\bar{m}}^{p}}{\partial \bar{\xi}^{p}}=-\overline{\bar{K}}^{p} \overline{\bar{\xi}}^{p}$
\end{tabular}

Tab. 2: State equations of the system.

\subsubsection{Equations of evolution of the internal variables}

Now, we determine the equations that govern the evolution of the internal variables of this concrete phenomenological constitutive law. We assume that the evolution is driven by the principle of maximum dissipation. With the state equations in Tab. 2 at hand we first rewrite the dissipation - Eqs. (5) and (6) - 
as:

$$
\begin{aligned}
& \dot{\mathcal{D}}=\underbrace{\sigma^{v} \dot{\bar{\epsilon}}^{v}}_{\dot{\overline{\mathcal{D}}}^{v}}+\underbrace{\sigma \dot{\bar{\epsilon}}^{p}+\bar{q}^{p} \dot{\bar{\xi}}^{p}+\bar{\kappa}^{p} \dot{\bar{\lambda}}^{p}}_{\dot{\overline{\mathcal{D}}}^{p}}+\underbrace{\frac{1}{2} \sigma \dot{\bar{D}} \sigma+\bar{q}^{p} \dot{\bar{\xi}}^{d}}_{\dot{\overline{\mathcal{D}}}^{d}}+ \\
& \underbrace{\left(\mathrm{t} \dot{\bar{u}}^{p}+\overline{\bar{q}}^{p} \dot{\overline{\bar{\xi}}}^{p}\right)}_{\dot{\overline{\mathcal{D}}}^{p}} \delta_{\bar{x}}^{(\frac{1}{2} \mathrm{t} \dot{\overline{\bar{D}}} \mathrm{t}+\underbrace{d}_{\overline{\overline{\mathcal{D}}}^{d}} \dot{\overline{\bar{\xi}}}^{d})} \delta_{\bar{x}}
\end{aligned}
$$

To characterize the evolution of the system, except the viscous one, we extend the principle of maximum dissipation - which was first formulated for plasticity problems $[14,21]$ - to our model and thus compute the flow of the internal variables by assuming that they maximize the dissipation. Remembering that the criteria functions in Eqs. (3) and (4) must be satisfied, we thus have to solve a problem of constrained maximization that involves inequalities. Denoting $f=\left(\sigma, \mathrm{t}, q^{p}, \kappa^{p}, q^{d}, \overline{\bar{q}}^{p}, \overline{\bar{q}}^{d}\right)$, we have the following problem $\mathcal{P}$ :

$\max _{f} \dot{\mathcal{D}}$ with constraints $\phi^{p}(f) \leq 0, \phi^{d}(f) \leq 0, \overline{\bar{\phi}}^{p}(f) \leq 0, \overline{\bar{\phi}}^{d}(f) \leq 0$

Such a problem can be solved with the Lagrange's multipliers method [15, 34]. We define the Lagrangian as:

$$
\mathcal{L}(f, \dot{\gamma})=\dot{\mathcal{D}}-\left(\dot{\gamma}^{p} \phi^{p}+\dot{\gamma}^{d} \phi^{p}+\dot{\overline{\bar{\gamma}}} \overline{\bar{\phi}}^{p}+\dot{\bar{\gamma}}^{d} \overline{\bar{\phi}}^{d}\right)
$$

where $\dot{\gamma}=\left(\dot{\gamma}^{p}, \dot{\gamma}^{d}, \dot{\bar{\gamma}}^{p}, \dot{\bar{\gamma}}^{d}\right)$ is the set of Lagrange's multipliers associated to each constraint. According to the Kuhn-Tucker conditions, if the set $f^{*}=\left\{f_{i}^{*}\right\}$ is a solution of $\mathcal{P}$, then there exists a unique set of Lagrange multipliers $\dot{\gamma}^{*}=\left\{\dot{\gamma}_{j}^{*}\right\}$ such that the following relations are verified for all $i$ and for all $j$ :

$$
\frac{\partial \mathcal{L}\left(f^{*}, \dot{\gamma}^{*}\right)}{\partial f_{i}}=0 \quad, \quad \frac{\partial \mathcal{L}\left(f^{*}, \dot{\gamma}^{*}\right)}{\partial \dot{\gamma}_{j}} \geq 0 \quad, \quad \dot{\gamma}_{j}^{*} \geq 0 \quad, \quad \dot{\gamma}_{j}^{*} \phi_{j}\left(f^{*}\right)=0
$$

where the last three conditions are referred to as the loading / unloading conditions. Finally, the equations of evolution of the internal variables are given in Tab. 3.

For the viscous internal variable $\bar{\epsilon}^{v}$, the evolution is expressed, in an associative form too, à la Perzyna [29]:

$$
\dot{\bar{\epsilon}}^{v}=\frac{\bar{\phi}^{v}}{\eta} \frac{\partial \bar{\phi}^{v}}{\partial \sigma}
$$

where $\eta$ is the viscous parameter assumed here to be rate-insensitive although this might not be the case [28]. Note that Eq. (14) is in accordance with the rheological relation in a dashpot $\sigma^{v}=\eta \dot{\epsilon}^{v}$.

Note that concrete is in general not an associative material. However, in the $1 \mathrm{D}$ context of this paper, we nevertheless assume that it is the case. All the equations of evolution of the internal variables thus are expressed in an associative form. The normality of the flow rule to the loading surface is in particular implied by the appeal to the maximum dissipation principle. 


\begin{tabular}{|c|c|}
\hline Continuous plasticity & Continuous damage \\
\hline $\begin{array}{l}\dot{\epsilon}^{p}=\dot{\gamma}^{p} \frac{\partial \phi^{p}}{\partial \sigma} \\
\dot{\xi}^{p}=\dot{\gamma}^{p} \frac{\partial \phi^{p}}{\partial q^{p}} \\
\dot{\lambda}^{p}=\dot{\gamma}^{p} \frac{\partial \phi^{p}}{\partial \kappa^{p}} \\
\dot{\gamma}^{p} \geq 0, \phi^{p} \leq 0, \dot{\gamma}^{p} \phi^{p}=0\end{array}$ & $\begin{array}{l}\dot{D} \sigma=\dot{\gamma}^{d} \frac{\partial \phi^{d}}{\partial \sigma} \\
\dot{\xi}^{d}=\dot{\gamma}^{d} \frac{\partial \phi^{d}}{\partial q^{d}} \\
\dot{\gamma}^{d} \geq 0, \phi^{d} \leq 0, \dot{\gamma}^{d} \phi^{d}=0\end{array}$ \\
\hline Discrete plasticity & Discrete damage \\
\hline $\begin{array}{l}\dot{\overline{\bar{u}}} p=\dot{\overline{\bar{\gamma}}} p \frac{\partial \overline{\bar{\phi}}^{p}}{\partial \mathrm{t}} \\
\dot{\overline{\bar{\xi}}}^{p}=\dot{\overline{\bar{\gamma}}} p \frac{\partial \overline{\bar{\phi}}^{p}}{\partial \overline{\bar{q}}^{p}} \\
\dot{\overline{\bar{\gamma}}}^{p} \geq 0, \overline{\bar{\phi}}^{p} \leq 0, \quad \dot{\bar{\gamma}}^{p} \overline{\bar{\phi}}^{p}=0\end{array}$ & $\begin{array}{l}\dot{\overline{\bar{D}}} \mathrm{t}=\dot{\overline{\bar{\gamma}}}^{d} \frac{\partial \overline{\bar{\phi}}^{d}}{\frac{\partial \mathrm{t}}{\overline{\bar{y}}^{\mathrm{t}}}} \\
\dot{\overline{\bar{\xi}}}^{d}=\dot{\overline{\bar{\gamma}}}^{d} \frac{\partial \overline{\bar{\phi}}^{d}}{\partial \overline{\bar{q}}^{d}} \\
\dot{\overline{\bar{\gamma}}}^{d} \geq 0, \overline{\bar{\phi}}^{d} \leq 0, \quad \dot{\bar{\gamma}}^{d} \overline{\bar{\phi}}^{d}=0\end{array}$ \\
\hline
\end{tabular}

Tab. 3: Equations of evolution of the internal variables.

\section{Generalized standard materials}

Materials that belong to the generalized standard materials class benefit from nice local and global stability properties required for robust implementation in, for instance, finite element procedures. For other materials, some attention must be paid on the local or global stability properties. There are five conditions, in an isothermal context, to be fulfilled for a material to enter the generalized standard materials class [24]:

1. Its Helmholtz free energy $\psi(\boldsymbol{\alpha})$ is convex in $\boldsymbol{\alpha}=\left\{\alpha_{i}\right\}$.

2. The laws of state for the internal variables are derived from the Helmholtz free energy that plays the role of a thermodynamic potential: $\mathcal{A}_{i}=-\frac{\partial \psi}{\partial \alpha_{i}}$.

3. The intrinsic dissipation verifies: $\dot{\mathcal{D}}=\sum \mathcal{A}_{i} \dot{\alpha}_{i} \geq 0$.

4. There exists a closed convex set $\mathcal{K}$ in the space of the associated variables $\mathcal{A}$ and $\mathcal{A} \in \mathcal{K}$.

5. The flux of the internal variables $\alpha_{i}$ is normal to the boundary of $\mathcal{K}$ (normality law).

As shown in section 2.2, the material model presented in this paper satisfies the conditions 2) and 5). The sets $\mathcal{K}$ mentioned in the condition 4) are characterized according to the criteria functions $\phi(\mathcal{A})$ introduced in Eq. (3) and (4) and their boundary is defined by the equations $\phi(\mathcal{A})=0$. In our $1 \mathrm{D}$ context, these criteria functions clearly define closed and convex sets in $\mathcal{A}$-space. About condition 1), we recall that a function of the form $f(x)=c x^{2}$ is convex only if $c>0$. The convexity of the Helmholtz free energy is therefore not verified for a negative phenomenological coefficient $\overline{\bar{K}}$, which is required to represent softening. 
Let prove that our model satisfies the condition 3). First, since $\eta>0$, $\dot{\overline{\mathcal{D}}}^{v}=\sigma \dot{\bar{\epsilon}}^{v}=\eta \dot{\bar{\epsilon}}^{v 2} \geq 0$. For the other dissipative mechanisms:

$$
\begin{aligned}
\dot{\mathcal{D}}^{\diamond} & =\quad \sigma \dot{\epsilon}^{\diamond}+q^{\diamond} \dot{\xi}^{\diamond} & & \\
& =\dot{\gamma}^{\diamond}\left(\sigma \frac{\partial \phi^{\diamond}}{\partial \sigma}+q^{\diamond}\right) & & \text { (see Tab. (3)) } \\
& =\dot{\gamma}^{\diamond}\left(\phi^{\diamond}+\sigma_{y, f, u}\right) & & \text { (see Eqs. (3) and (4)) }
\end{aligned}
$$

According to the loading / unloading conditions Eq. (13), $\dot{\gamma}^{\diamond} \geq 0$ and $\dot{\gamma}^{\diamond} \phi^{\diamond}=0$. Moreover, the material parameters verifies $\sigma_{y, f, u}>0$ and thus, finally, $\dot{\mathcal{D}}^{\diamond} \geq 0$. Fulfillment of the condition 3 ) ensures local stability properties (hysteretic loops builded clockwise).

To conclude this section, (i) because condition 1) is not satisfied with $\overline{\bar{K}}<0$, the global stability of the numerical model has to be investigated: the tangent stiffness matrix could become non-invertible during nonlinear resolution procedure; moreover, (ii) the constitutive model presented here is rate-sensitive and the existence of a critical time-step must thus also be investigated; finally, (iii) $\mathcal{A}$-space is divided into two parts, one for tension and the other for compression, which shall be considered in the numerical resolution phase. In this paper, we only focus on the physical formulation of the model, but it can be shown that these three points can be treated in an efficient way that makes the numerical implementation in finite element procedures robust at local and global levels.

\section{Euler-Lagrange equations of a concrete 1D structure in dynamic loading}

In this section, we move up from the formulation of the local constitutive model to the formulation of the structural mechanical problem. This latter, illustrated here in the $1 \mathrm{D}$ case, has to be adapted to the local constitutive model and requires the definition of an enhanced displacement field.

\subsection{Enhanced displacement field kinematics}

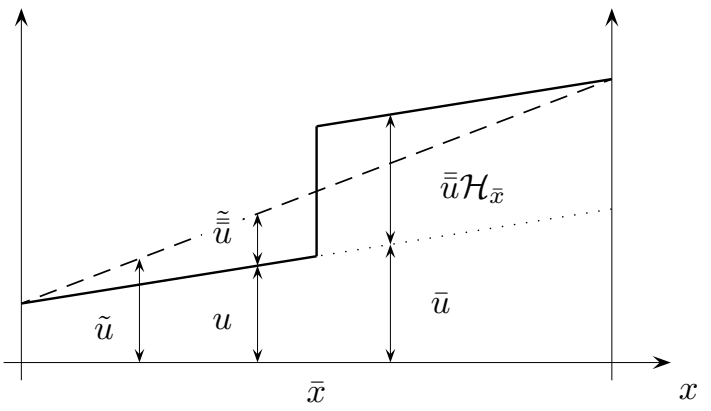

Fig. 2: Enhanced displacement field in a $1 \mathrm{D}$ structure. The solid, dashed and dotted lines represent the enriched displacement field $u(x, t)$, the smooth displacement field $\tilde{u}(x, t)$ and the continuum displacement field $\bar{u}(x, t)$.

As shown in Fig. 2, the displacement field is written as the sum of a smooth linear part $\tilde{u}(x, t)$ enhanced by an additional part $\tilde{\bar{u}}(x, t)$ to take into account 
the possible appearance of a displacement jump $\overline{\bar{u}}(t)$ in the 1D structure $[10,16]$ :

$$
u(x, t)=\tilde{u}(x, t)+\underbrace{\left(\mathcal{H}_{\bar{x}}(x)-\varphi(x)\right) \overline{\bar{u}}(t)}_{\tilde{\bar{u}}(x, t)}
$$

which can also be written, in the form adapted for identification with Eq. (1), as:

$$
u(x, t)=\underbrace{\tilde{u}(x, t)-\varphi(x) \overline{\bar{u}}(t)}_{\bar{u}(x, t)}+\overline{\bar{u}}(t) \mathcal{H}_{\bar{x}}(x)
$$

Then $\epsilon(x, t)=\bar{\epsilon}(x, t)+\overline{\bar{u}}(t) \delta_{\bar{x}}$ with:

$$
\bar{\epsilon}(x, t)=\frac{\partial \bar{u}(x, t)}{\partial x}=\frac{\partial \tilde{u}(x, t)}{\partial x}-\frac{d \varphi(x)}{d x} \overline{\bar{u}}(t)
$$

In this $1 \mathrm{D}$ context, only the failure mode $\mathrm{I}$ is considered; we refer to [9] for a presentation of the kinematics with embedded crack of quadrilateral $2 \mathrm{D}$ finite elements where several failure modes are considered.

\subsection{Complementary Lagrangian variational formulation}

The kinetic energy, assumed to depend only on the smooth part of the displacement, is:

$$
T(\tilde{u}, t)=\frac{1}{2} \int_{\Omega} \rho\left(\frac{\partial \tilde{u}(x, t)}{\partial t}\right)^{2} d \Omega
$$

where the volumic mass $\rho$ in the volume $\Omega$ is considered as constant.

The total potential energy can be written:

$$
U(\tilde{u}, \overline{\bar{u}}, \boldsymbol{\alpha}, t)=\int_{\Omega} \psi(\tilde{u}, \overline{\bar{u}}, \boldsymbol{\alpha}, t) d \Omega-U^{e x t}(\tilde{u}, t)
$$

To introduce the stress fields $\sigma$ and $\mathrm{t}$ as other independent variables of the problem and thus give a more general setting that can be helpful to derive the governing equations of the problem $[22,32]$, we appeal to the partial Legendre transformation of the Helmholtz free energy:

$$
\begin{aligned}
& \psi^{+}=\sigma^{e} \bar{\epsilon}^{e}-\frac{1}{2} \sigma^{e} \bar{E}^{-1} \sigma^{e}+\left(\mathrm{t} \overline{\bar{u}}^{d}-\frac{1}{2} \mathrm{t} \overline{\bar{D}} \mathrm{t}+\overline{\bar{\Xi}}^{d}\right) \delta_{\bar{x}} \\
& \psi^{-}=\sigma^{d} \bar{\epsilon}^{d}-\frac{1}{2} \sigma^{d} \bar{D} \sigma^{d}+\bar{\Xi}^{p}+\bar{\Lambda}^{p}+\bar{\Xi}^{d}+\left(\mathrm{t} \overline{\bar{u}}^{e}-\frac{1}{2} \mathrm{t} \overline{\bar{E}}^{-1} \mathrm{t}+\bar{\Xi}^{p}\right) \delta_{\bar{x}}
\end{aligned}
$$

Recalling that $\bar{\epsilon}^{e}=\bar{\epsilon}^{v}$ in tension, $\bar{\epsilon}^{d}=\bar{\epsilon}^{v}$ in compression, $\sigma=\sigma^{v}+\sigma^{e / d}$ and according to Eqs. (1) and (18), we rewrite these equations with respect to $\tilde{u}(x, t)$, $\overline{\bar{u}}(t), \sigma(x, t), \mathrm{t}(t)$ and $\boldsymbol{\alpha}$ :

$$
\begin{aligned}
\psi^{+}= & \sigma\left(\frac{\partial \tilde{u}}{\partial x}-\frac{d \varphi}{d x} \overline{\bar{u}}-\bar{\epsilon}^{p}\right)-\sigma^{v} \bar{\epsilon}^{v}-\frac{1}{2}\left(\sigma-\sigma^{v}\right) \bar{E}^{-1}\left(\sigma-\sigma^{v}\right) \\
& +\left(\mathrm{t}\left(\overline{\bar{u}}-\overline{\bar{u}}^{p}\right)-\frac{1}{2} \mathrm{t} \overline{\bar{D}} \mathrm{t}+\overline{\bar{\Xi}}^{d}\right) \delta_{\bar{x}} \\
\psi^{-}= & \sigma\left(\frac{\partial \tilde{u}}{\partial x}-\frac{d \varphi}{d x} \overline{\bar{u}}-\bar{\epsilon}^{p}\right)-\sigma^{v} \bar{\epsilon}^{v}-\frac{1}{2}\left(\sigma-\sigma^{v}\right) \bar{D}\left(\sigma-\sigma^{v}\right) \\
& +\bar{\Xi}^{p}+\bar{\Lambda}^{p}+\bar{\Xi}^{d}+\left(\mathrm{t}\left(\overline{\bar{u}}-\overline{\bar{u}}^{p}\right)-\frac{1}{2} \mathrm{t} \overline{\bar{E}}^{-1} \mathrm{t}+\overline{\bar{\Xi}}^{p}\right) \delta_{\bar{x}}
\end{aligned}
$$


We denote $L$ the Lagrangian of the mechanical system. It is defined as $L=T-U$ and Lagrange's variational principle can be written as:

$$
\int_{t_{1}}^{t_{2}} \delta L(\tilde{u}, \overline{\bar{u}}, \sigma, \mathrm{t}, t) d t=0 \quad \forall t_{1}, \forall t_{2}
$$

with $\delta \tilde{u}(x, t), \delta \overline{\bar{u}}(t), \delta \sigma(x, t), \delta \mathrm{t}(t)$ null when $t=t_{1}$ and $t=t_{2}$.

After some calculus (integration by parts,...), we obtain the following set of governing equations of the system available for tension and compression:

$$
\begin{aligned}
& \int_{\Omega} \rho \frac{\partial^{2} \tilde{u}}{\partial t^{2}} \delta \tilde{u} d \Omega+\int_{\Omega} \frac{\partial \psi}{\partial \tilde{u}} \delta \tilde{u} d \Omega-\delta U^{e x t}=0 \\
& \int_{\Omega} \frac{\partial \psi}{\partial \overline{\bar{u}}} d \Omega \delta \overline{\bar{u}}=0 \\
& \int_{\Omega} \frac{\partial \psi}{\partial \sigma} \delta \sigma d \Omega=0 \\
& \int_{\Omega} \frac{\partial \psi}{\partial \mathrm{t}} d \Omega \delta \mathrm{t}=0
\end{aligned}
$$

which leads, with $\Gamma$ denoting the section in which the displacement is likely to occur and by remembering that neither $\overline{\bar{u}}$ nor $\mathrm{t}$ depends on the position $x$ (they are only defined at the position $x_{\Gamma}$ of the section $\Gamma$ ), to the set of Euler-Lagrange equations of a concrete 1D structure written in Tab. 4.

\begin{tabular}{ll}
\hline Tension & Compression \\
\hline $\int_{\Omega}\left(\rho \frac{\partial^{2} \tilde{u}}{\partial t^{2}} \delta \tilde{u}+\frac{\partial \delta \tilde{u}}{\partial x} \sigma\right) d \Omega-\delta U^{e x t}=0$ & $\int_{\Omega}\left(\rho \frac{\partial^{2} \tilde{u}}{\partial t^{2}} \delta \tilde{u}+\frac{\partial \delta \tilde{u}}{\partial x} \sigma\right) d \Omega-\delta U^{e x t}=0$ \\
$\int_{\Omega}\left(\frac{d \varphi}{d x} \sigma-\mathrm{t} \delta_{\bar{x}}\right) d \Omega \delta \overline{\bar{u}}=0$ & $\int_{\Omega}\left(\frac{d \varphi}{d x} \sigma-\mathrm{t} \delta_{\bar{x}}\right) d \Omega \delta \overline{\bar{u}}=0$ \\
$\int_{\Omega} \delta \sigma\left(\frac{\partial \tilde{u}}{\partial x}-\frac{d \varphi}{d x} \overline{\bar{u}}-\bar{\epsilon}^{v}-\bar{\epsilon}^{p}\right) d \Omega=0$ & $\int_{\Omega} \delta \sigma\left(\frac{\partial \tilde{u}}{\partial x}-\frac{d \varphi}{d x} \overline{\bar{u}}-\bar{\epsilon}^{v}-\bar{\epsilon}^{p}\right) d \Omega=0$ \\
$\int_{\Gamma}\left(\overline{\bar{u}}-\overline{\bar{u}}^{p}-\overline{\bar{D}} \mathrm{t}\right) d \Gamma \delta \mathrm{t}=0$ & $\int_{\Gamma}\left(\overline{\bar{u}}-\overline{\bar{u}}^{p}-\overline{\bar{E}}^{-1} \mathrm{t}\right) d \Gamma \delta \mathrm{t}=0$
\end{tabular}

Tab. 4: Euler-Lagrange equations of a concrete 1D structure.

Either for tension or compresssion, the first equation in Tab. 4 enforces satisfying global force equilibrium, the second equation gives the condition of compatibility between the continuum $\sigma$ and discrete $\mathrm{t}$ stresses, the third and fourth equations correspond to the weak form of the local continuum and discrete constitutive models.

To give another - also simplified in our viewpoint - expression of the set of the governing equations of the problem, one can take advantage of the dependancy between the continuum and the discrete stresses that appears in Tab. 4: $\int_{\Omega}\left(\frac{d \varphi}{d x} \sigma-\mathrm{t} \delta_{\bar{x}}\right) d \Omega=0 \Rightarrow \mathrm{t}=\mathrm{t}(\sigma)$. Then, the expression of the Lagrangian is 
modified according to:

$$
\begin{gathered}
\int_{\Omega} \psi^{+} d \Omega=\int_{\Omega}\left(\sigma\left(\frac{\partial \tilde{u}}{\partial x}-\bar{\epsilon}^{v}-\bar{\epsilon}^{p}\right)+\left(-\mathrm{t} \overline{\bar{u}}^{p}-\frac{1}{2} \mathrm{t} \overline{\bar{D}} \mathrm{t}+\overline{\bar{\Xi}}^{d}\right) \delta_{\bar{x}}\right) d \Omega \\
\int_{\Omega} \psi^{-} d \Omega=\int_{\Omega}\left(\sigma\left(\frac{\partial \tilde{u}}{\partial x}-\bar{\epsilon}^{v}-\bar{\epsilon}^{p}\right)+\bar{\Xi}^{p}+\bar{\Lambda}^{p}+\bar{\Xi}^{d}\right. \\
\left.+\left(-\mathrm{t} \overline{\bar{u}}^{p}-\frac{1}{2} \mathrm{t} \overline{\bar{E}}^{-1} \mathrm{t}+\overline{\bar{\Xi}}^{p}\right) \delta_{\bar{x}}\right) d \Omega
\end{gathered}
$$

Moreover, the response of the localization section is supposed to be infinitely rigid before the stress reaches an ultimate value and therefore $\overline{\bar{E}}^{-1} \rightarrow 0$. Finally, denoting $A_{\Gamma}$ the area of the section $\Gamma$ where displacement localization can take place, the simplified form of these equations is written in Tab. 5. Only two unknown fields remain: the smooth displacement $\tilde{u}(x, t)$ and the continuum stress $\sigma(x, t)$.

\begin{tabular}{ll}
\hline Tension & Compression \\
\hline $\int_{\Omega}\left(\rho \frac{\partial^{2} \tilde{u}}{\partial t^{2}} \delta \tilde{u}+\frac{\partial \delta \tilde{u}}{\partial x} \sigma\right) d \Omega-\delta U^{e x t}=0$ & $\int_{\Omega}\left(\rho \frac{\partial^{2} \tilde{u}}{\partial t^{2}} \delta \tilde{u}+\frac{\partial \delta \tilde{u}}{\partial x} \sigma\right) d \Omega-\delta U^{e x t}=0$ \\
$\int_{\Omega} \delta \sigma\left(\frac{\partial \tilde{u}}{\partial x}-\bar{\epsilon}^{v}-\bar{\epsilon}^{p}\right.$ & $\int_{\Omega} \delta \sigma\left(\frac{\partial \tilde{u}}{\partial x}-\bar{\epsilon}^{v}-\bar{\epsilon}^{p}-\overline{\bar{u}}^{p} \frac{d \mathrm{t}}{d \sigma} \delta_{\bar{x}}\right) d \Omega=0$ \\
$\left.-\left(\overline{\bar{u}}^{p}+\overline{\bar{D}} \mathrm{t}\right) \frac{d \mathrm{t}}{d \sigma} \delta_{\bar{x}}\right) d \Omega=0$ & \\
with $\quad \mathrm{t}=\mathrm{t}(\sigma)=\frac{1}{A_{\Gamma}} \int_{\Omega} \frac{d \varphi}{d x} \sigma d \Omega$ & with $\quad \mathrm{t}=\mathrm{t}(\sigma)=\frac{1}{A_{\Gamma}} \int_{\Omega} \frac{d \varphi}{d x} \sigma d \Omega$
\end{tabular}

Tab. 5: Final Euler-Lagrange equations of a concrete 1D structure.

\section{Numerical applications}

The numerical implementation has been done in the general purpose finite element computer program FEAP [35]. Because any external distributed loading is involved (self-weight is in particular neglected) in these applications, the concrete structure is always modeled with constant-stress bar elements. The 1D structure tested is presented in Fig. 3.

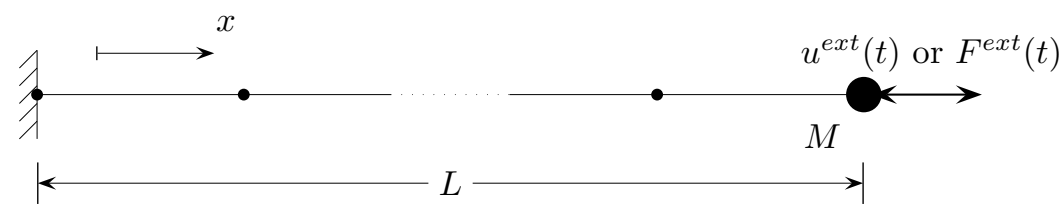

Fig. 3: 1D concrete structure model used for the numerical applications. The cross-section area $S=0.04 \mathrm{~m}^{2}$, the length $L=1 \mathrm{~m}$, the added mass $M=7.0 e^{5} \mathrm{~kg}$, the volumic mass $\rho=2400 \mathrm{~kg} \cdot \mathrm{m}^{-3}$ and the concrete elastic modulus $E=35 \mathrm{GPa}$.

Neglecting the concrete mass $m=\rho A L$ as compared to the added mass $M$, we compute the stiffness $k$, the fundamental pulsation $\omega$ and period $T$ of this 
structure as:

$$
k=\frac{E S}{L}=1.4 \mathrm{GPa} \quad ; \quad \omega=\sqrt{\frac{k}{M}}=44.7 \mathrm{~s}^{-1} \quad ; \quad T=\frac{2 \pi}{\omega}=0.14 \mathrm{~s}
$$

\subsection{Identification of a concrete law}

All the parameters of the $1 \mathrm{D}$ cyclic concrete model presented in this paper have a clear physical meaning. It is shown in this section that (i) the viscous parameter $\eta$ is related to the material critical damping ratio $\xi$, (ii) the tensile softening law parameter $a$ is related to the fracture energy $G_{F}$ and (iii) all the other parameters can be directly identified from the experimental stress-strain response of a concrete specimen in quasi-static cyclic loading.

\subsubsection{Identification of the viscous parameter $\eta$}

We show here that the viscous parameter $\eta$ can be interpreted as a material property that can be identified according to, among others, the experimental results of free low-amplitude - so that the structure remains elastic - vibration tests (Fig. 4):

1. First, denoting $\xi$ the critical damping ratio and $c$ the structural viscous parameter, if one assumes that $\xi<<1$, one can write [5]:

$$
c=2 \xi m \omega
$$

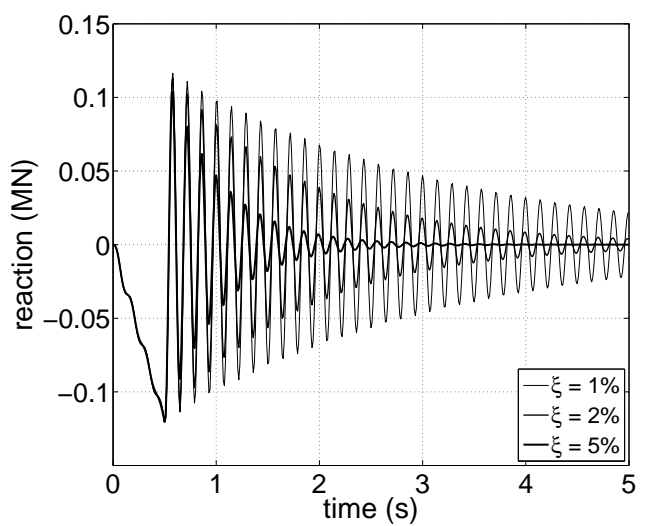

Fig. 4: Free-vibrations viscoelastic response of the tested structure for various critical damping ratios.

2. Then we link the material viscous parameter $\eta$ to the structural one $c$ starting from the 1D local form of the equilibrium and the Kelvin-Voigt model constitutive equations:

$$
\rho \ddot{u}+\frac{\partial \sigma}{\partial x}=0 \quad ; \quad \sigma=E \frac{\partial u}{\partial x}+\eta \frac{d}{d t}\left(\frac{\partial u}{\partial x}\right)
$$


For a tested structure with constant cross-section area $S$ and strain field $\left(\frac{\partial u}{\partial x}=\frac{u}{L}\right)$, the introduction of Eq. $(28)_{2}$ into Eq. $(28)_{1}$ and then the integration of the resulting local equation over the whole structure gives:

$$
\underbrace{\rho S L}_{m} \ddot{u}+\underbrace{\frac{S}{L} \eta}_{c} \dot{u}+\underbrace{\frac{E S}{L}}_{k} u=0
$$

3. The Kelvin-Voigt viscoelastic model exhibits a hysteretic - energy dissipative - behavior in cyclic loading as illustrated in Fig. 5 for an excitation of the form $\bar{u}=A \cdot \sin (\Omega t)$ with, except for other indicated values, $A=0.3 \mathrm{~mm}, \Omega=157.08 \mathrm{~s}^{-1}$ and $\tau=0.001 \mathrm{~s}$. The amount of energy dissipated per cycle $D^{c y c}$ - the area of the elliptical loop - can be analytically written as [36]:

$$
D^{c y c}=\pi c \Omega A^{2}
$$

The main drawback of the Kelvin-Voigt model is that $D^{c y c}$ is dependent on the loading frequency $\Omega$. It is indeed not realistic in particular for seismic excitations [36]. This dependency is only negligeable in the cases where the response is primarily represented by the resonant pulsation, that is $\Omega \simeq \omega$.
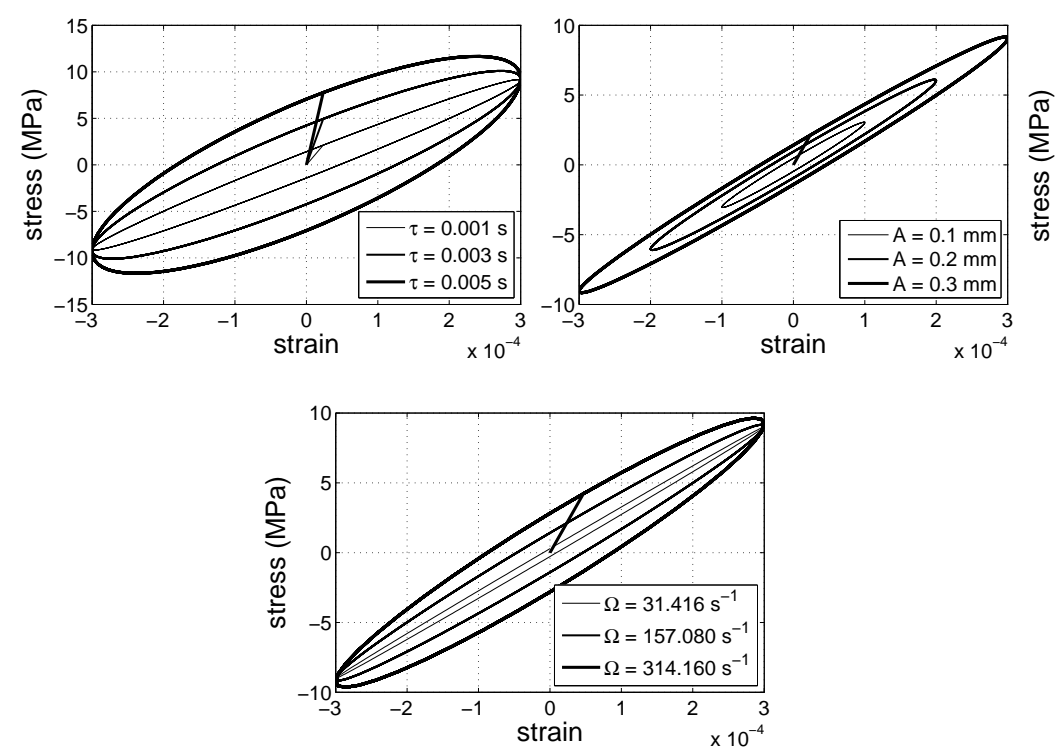

Fig. 5: Hysteretic response of the viscoelastic model in quasi-static sine loading for several [top-left] viscous parameters, [top-right] loading amplitudes and [bottom] forcing pulsations.

4. Now, suppose that a concrete structure is excited by an impulsion that is weak enough not to active the nonlinear phenomena that are represented 
by our model, and that, assuming that the response is primarily represented by the fundamental pulsation $\omega$, a critical damping ratio $\xi=1 \%$ is measured (for instance thanks to the logarithmic decrement), we can then see $\eta$ as a material property and identify it according to the following relation:

$$
\eta=\frac{2 \xi m \omega L}{S}
$$

In the case of a tested structure identical to those in Fig. 3, we compute:

$$
\eta=15.7 \mathrm{MPa} . \mathrm{s} \quad ; \quad \tau=\frac{\eta}{E} \simeq 4.5 e^{-4} \mathrm{~s}
$$

\subsubsection{Identification of the tensile softening parameter $a$}

The tensile softening parameter $a$ can be computed according to the fracture energy $G_{F} . G_{F}$ is one of the parameters that characterize the softening stressseparation (crack opening) curve of the cohesive crack model [13, 3] and represents the total amount of energy that has to be furnished in tension to a section between the time $t_{l o c}$ when displacement begins to localize and the time $t_{c r i}$ that we define as the time when the local softening problem becomes (numerically) ill-posed, because $\overline{\bar{K}}^{d}<0$; physically, $t_{c r i}$ has to coincide with the time when the section is completely broken: when all the energy $G_{F}$ is consumed. In the case of our concrete model, one can demonstrate that $t_{c r i}$ is always defined and that:

$$
G_{F}=\int_{t_{l o c}}^{t_{c r i}} \mathrm{t} \dot{\bar{u}} d t=\frac{\sigma_{u}^{t}}{2 a} \Rightarrow a=\frac{\sigma_{u}^{t}}{2 G_{F}}
$$

In addition, there exists an empirical relation [2] that allows computing $G_{F}$ according to material characteristics:

$$
G_{F}=2.5 \alpha_{0}\left(\frac{\sigma_{u}^{c}}{0.051}\right)^{0.46}\left(1+\frac{d_{a}}{11.27}\right)^{0.22}\left(\frac{w}{c}\right)^{-0.30}
$$

with $\sigma_{u}^{c}$ in MPa and where $\alpha_{0}=1$ for rounded aggregates, $\alpha_{0}=1.44$ for crushed or angular aggregates, $d_{a}$ is the maximum aggregate size in $\mathrm{mm}$ and $\frac{w}{c}$ is the water-cement ratio. Finally, for a concrete with $\alpha_{0}=1.44, d_{a}=25 \mathrm{~mm}$ and

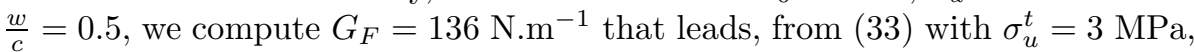
to:

$$
a=22060 \mathrm{~m}^{-1}
$$

\subsubsection{Identification of the other parameters}

Here is the procedure to identify the remaining parameters of the model from the stress-strain experimental response in Fig. 1 [left]:

1. Identify the Young's modulus for tensile and compressive parts. We consider here that they have the same value: $E=\bar{E}=35 \mathrm{GPa}$.

2. Identify the set of stresses $\sigma_{y}, \sigma_{f}, \sigma_{u}^{c}, \sigma_{u}^{t}$ so as to characterize the changes of the slope of the backbone curve. We choose: $\sigma_{y}=3 \mathrm{MPa}, \sigma_{f}=37 \mathrm{MPa}$, $\sigma_{u}^{c}=49 \mathrm{MPa}$ and $\sigma_{u}^{t}=0.6 \cdot \sigma_{u}^{c}=3 \mathrm{MPa}$. 
3. Identify simultaneously $\bar{K}^{p}$ and $\bar{H}^{p}$ so as they describe both the strain hardening phase of the backbone curve in the range $\sigma_{y}<\sigma<\sigma_{f}$ and the shape of the local hysteresis loops.

4. Identify $\bar{K}^{d}$ so as to describe the remaining strain hardening phase of the backbone curve in the range $\sigma_{f}<\sigma<\sigma_{u}^{c}$.

5. Identify $\overline{\bar{K}}^{p}$ so as to describe the softening part of the backbone curve.

Fig. 1 [right] is plotted with the parameters indicated above. Note that although all the parameters have a clear physical meaning, they also are all connected and the results of a first identification process thus often need to be refined. Indeed, the slope of the hardening part of the backbone curve in the range $\sigma_{y}<\sigma<\sigma_{f}$ is $C_{1}=\frac{\bar{E} \bar{K}^{p}}{E+K^{p}}$, in the range $\sigma_{f}<\sigma<\sigma_{u}^{c}$ is $C_{2}=\frac{C_{1} \bar{K}^{d}}{C_{1}+\bar{K}^{d}}$ and the slope of the softening part of the backbone curve is $C_{3}=\frac{C_{2} \bar{K}^{p}}{C_{2}+\bar{K}^{p}}$. However, in the absence of an automatic identification process [19], following the procedure indicated above gives satisfying results within a few iterations. Note also that, although we observed that activating plasticity before damage leads to a better identification, it is possible to invert the roles of $\sigma_{y}$ and $\sigma_{f}$.

\subsection{Mesh objectivity}

In finite element procedures, when displacement localizes, the solution of the mechanical problem can depend on the size of the mesh. In the formulation of our model, we introduced a strong discontinuity - a displacement jump - [16, 33] and thus concentrated the localization in a zero-length zone. This method leads to a formulation that does not need any characteristic length and thus satisfies the mesh objectivity requirement, that is unicity of the solution, in a bar as illustrated in Fig. 6 where the quasi-static tensile response of the section where displacement localizes in the bar in Fig. 3 submitted to an imposed displacement is plotted.

Concerning the modeling of the tensile softening phase, the fracture energy is so small with resect to the elastic energy that it is numerically difficult to grasp the post peak tensile response of concrete bar. The curve in Fig. 6 has been plotted with 4,000 points, which cannot be achieved for instance in seismic applications. Note that the presence of viscosity helps the numerical representation of this tensile softening part.

\subsection{Loading rate-sensitive response}

The four main effects of the loading strain-rate on the response of concrete are: the increases of (i) the compressive strength [4, 20], (ii) the tensile strength [37], (iii) the Young's modulus [37] and (iv) the brittle behavior [7]. For seismic excitations, the strain rates are comprised between $10^{-5} \mathrm{~s}^{-1}$ and $10^{-2} \mathrm{~s}^{-1}$, which respectively corresponds to an increase of (i) the compression strength between 0 and $30 \%$ [4, 20], (ii) the tensile strength between 0 and $60 \%$ [37] and (iii) the Young's modulus between 0 and 10\% [37].

The numerical applications presented in Fig.7 [left] show that the Young's modulus computed for a loading strain rate of $10^{-2} \mathrm{~s}^{-1}$ is about $5.7 \%$ larger than the one computed for a loading strain rate of $10^{-5} \mathrm{~s}^{-1}$. The increase of 


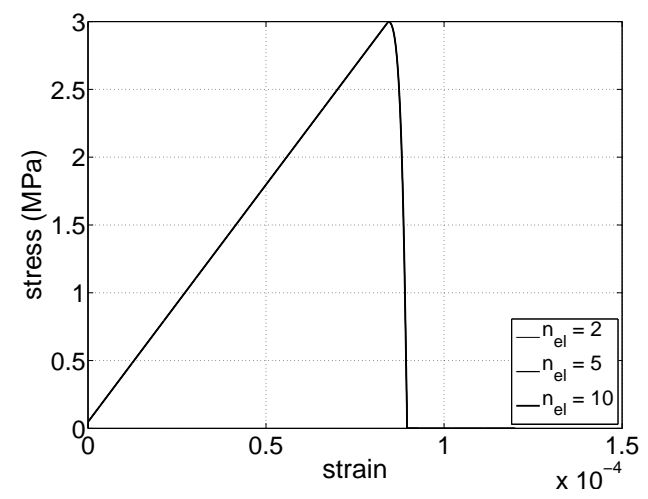

Fig. 6: Tensile response of the section where the discontinuity appears. $n_{e l}$ is the number of elements of the regular mesh of the bar.

Young's modulus is thus represented by the model but a little underestimated here. However, this increase is related to the viscous parameter $\tau$ computed in these numerical applications from the damping ratio $\xi=1 \%$ whose value has been arbitrarily chosen: one can thus hope that, in reality, the right value for the damping ratio would lead to a correct modeling of the increase of elastic stiffness. The compressive response is presented in Fig.7 [right]. The proposed concrete model has not been developed yet to represent by itself the rate-dependent tensile and compressive strengths, what can be done a priori by hand, as it is common practice in engineering where the parameters that define the strengths for concrete in quasi-static loading are majorated for seismis analyses.
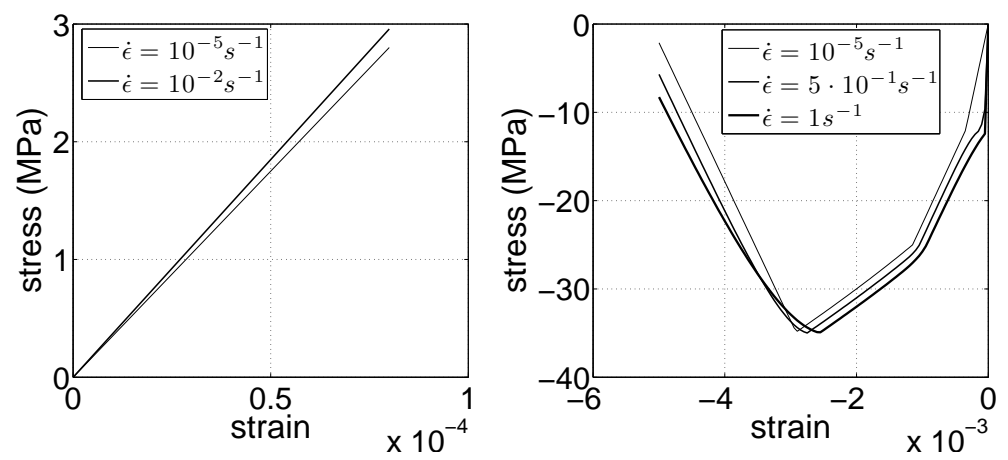

Fig. 7: Tensile [left] and [right] compressive concrete response for several loading rates. 


\subsection{Seismic application}

\subsubsection{Global seismic response}

We focus on the compressive part only of the concrete law in Fig. 1. Thus a static compressive normal force in imposed to the bar before the seimic excitation. In the absence of steel, once the strength degradation process has begun, the structure is no more capable of resisting the seismic force demand and global equilibrium can no more be satisfied; we therefore couple to the concrete bar an elastic steel bar with cross section area $S_{s}=0.02 \cdot S_{c}=8 e^{-4} \mathrm{~m}^{2}$ and Young's modulus $E_{s}=210 \mathrm{GPa}$. The behavior law for concrete is those presented in Fig. 1 [right]. The loading pattern is shown in Fig. 8 and the global structural response in Fig. 9.

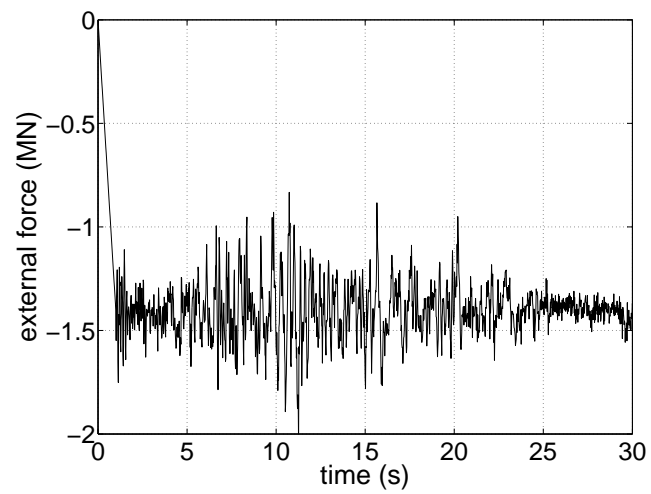

Fig. 8: Loading pattern: static loading + seismic loading time histories.
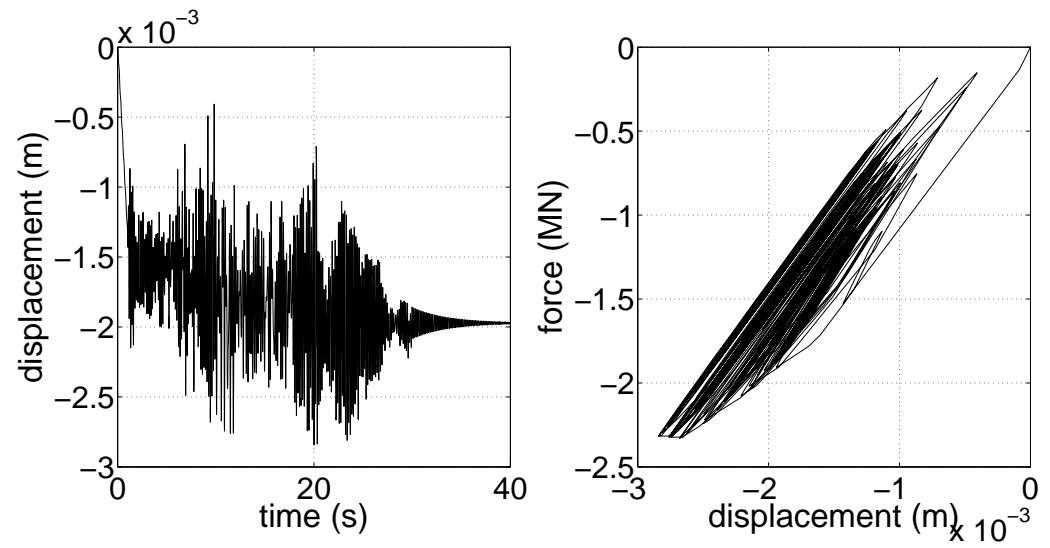

Fig. 9: [left] Right-node displacement and [right] reaction-displacement timehistories. 


\subsubsection{Local response and intrinsic dissipated energies}

$T I V$ provides a useful framework to quantify the material intrinsic dissipation. Indeed, the amount of continuum viscous, plastic and damage energies dissipated in all the structure wide and the discrete plastic and damage energies dissipated in the section where displacement localizes can be computed according to Eq. (10):

$$
\begin{aligned}
& \bar{E}^{v}=\int_{0}^{T} \int_{\Omega} \dot{\overline{\mathcal{D}}}^{v} d \Omega d t=\int_{0}^{T} \int_{\Omega} \sigma^{v} \dot{\bar{\epsilon}}^{v} d \Omega d t \\
& \bar{E}^{p}=\int_{0}^{T} \int_{\Omega} \dot{\overline{\mathcal{D}}}^{p} d \Omega d t=\int_{0}^{T} \int_{\Omega}\left(\sigma \dot{\bar{\epsilon}}^{p}+\bar{q}^{p} \dot{\bar{\xi}}^{p}+\bar{\kappa}^{p} \dot{\bar{\lambda}}^{p}\right) d \Omega d t \\
& \bar{E}^{d}=\int_{0}^{T} \int_{\Omega} \dot{\overline{\mathcal{D}}}^{d} d \Omega d t=\int_{0}^{T} \int_{\Omega}\left(\frac{1}{2} \sigma \dot{\bar{D}} \sigma+\bar{q}^{p} \dot{\bar{\xi}}^{d}\right) d \Omega d t \\
& \overline{\bar{E}}^{p}=\int_{0}^{T} \int_{\Omega} \dot{\overline{\overline{\mathcal{D}}}}^{p} d \Omega d t=\int_{0}^{T} \int_{\Gamma}\left(\mathrm{t} \dot{\bar{u}}^{p}+\overline{\bar{q}}^{p} \dot{\overline{\bar{\xi}}}^{p}\right) d \Gamma d t \\
& \overline{\bar{E}}^{d}=\int_{0}^{T} \int_{\Omega} \dot{\overline{\bar{D}}}^{d} d \Omega d t=\int_{0}^{T} \int_{\Gamma}\left(\frac{1}{2} \mathrm{t} \dot{\overline{\bar{D}}} \mathrm{t}+\overline{\bar{q}}^{d} \dot{\overline{\bar{\xi}}}^{d}\right) d \Gamma d t
\end{aligned}
$$

Note that the total intrinsic dissipation is composed by a volumic and a surfacic - in the section $\Gamma$ where displacement localizes - part.

The local response of material points located outside and inside $\Gamma$ is ploted in Fig 10: the localization of the displacement in $\Gamma$ leads to a larger amount of dissipated energy in this section. The amount and sources of intrinsic energy dissipation within the seimic excitation are detailed in Fig. 11. Around $t=11 \mathrm{~s}$, a big amount of seimic energy is imparted to the structure (see Fig. 8); so as this latter do not collapse, it has to dissipate this input energy. The input energy is converted both into stored energy in the material and dissipated energy (irreversible mechanisms).
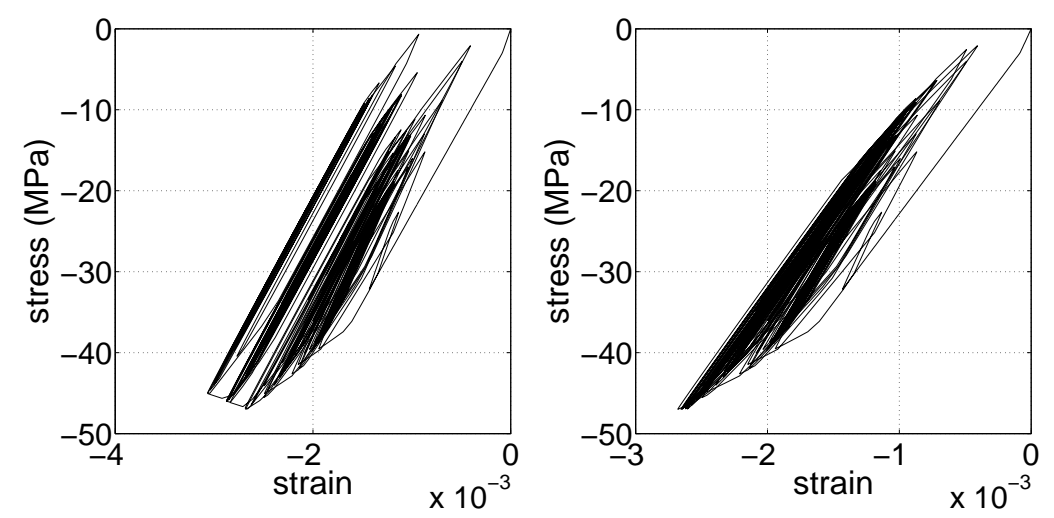

Fig. 10: Local response of a material point [left] located and [right] not located in section $\Gamma$ where displacement localizes. 


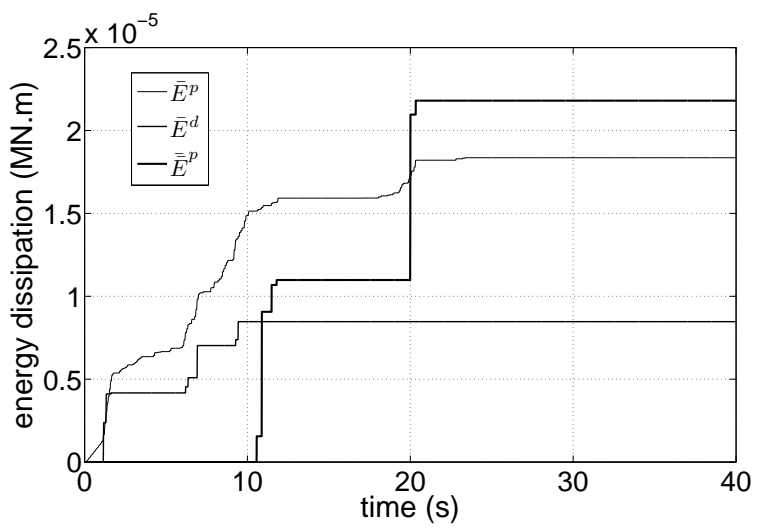

Fig. 11: Continuum plastic and damage and discrete plastic intrinsic dissipated energy time-histories.

\section{Conclusion and future work}

In this paper, the theoretical formulation of a 1D cyclic constitutive concrete model is presented. The model is capable of representing most of the salient phenomena that can be experimentally observed in the stress-strain response of a concrete representative elementary volume (REV) - at macroscale - in cyclic 1D loading: brittle loading-rate dependent behavior in tension, quasibrittle loading-rate dependent behavior in compression with strain hardening, appearance of residual deformation, stiffness and strength degradations, local hysteretis. Although the details of the numerical implementation are not given in this paper, the model has been developed to be implemented in finite element numerical procedures and thus special attention is paid on its robustness: (i) the conditions under which the model enters the generalized standard materials class are determined and (ii) linear hardening / softening laws that make the resolution processes noniterative at the local - or numerical integration point - level are prefered to nonlinear ones. The Euler-Lagrange equations of a 1D structure, made of this concrete material, in dynamic loading are derived and finally some numerical applications are presented. They illustrate that: the viscous parameter can be considered as a material property, the objectivity of the mesh of the tested bar is satisfied when displacement localizes and the material REV thus exhibits a softening behavior, the model can represent the salient phenomena that occur in concrete in cyclic loading.

The model is developed in the framework of thermodynamics with internal variables, that is that a set of internal variables is chosen to represent the experimentally observed response of a concrete REV in 1D cyclic loading. All the material parameters introduced have a clear physical meaning, what makes their identification simple. The model is developed in a purely phenomenological way at macroscale: for instance "strain hardening" means here "observed increase of the yield stress while strain increases" and has no relation with physical effects that occur at lower scales such as dislocations motion well known for steel materials. One could give a more refined description of the micro- or even 
nanoscopic behavior of concrete and one could hope that relating physical microor nanoscopic effects to the macroscopic response of a concrete REV would still improve the accuracy of the material models. However, our approach, albeit phenomenological and thus somehow arbitrary, can be efficiently used in common computer programs for large-scale structural numerical dynamic analyses. To that purpose, the implementation of the model in a fiber beam element is currently being achieved.

\section{Acknowledgements}

The financial support provided by the French Ministry of Research, the Quebec Fund for Research on Nature and Technology (FQRNT), the Natural Science and Engineering Research Council of Canada (NSERC), and ENS-Cachan Invited Professor Funds is acknowledged.

\section{References}

[1] Bhattacharjee SS, Léger P. Seismic cracking and energy dissipation in concrete gravity dams. Earthquake Engineering and Structural Dynamics 1993; 22(11): 991-1007.

[2] Bazant ZP, Becq-Giraudon E. Statistical prediction of fracture parameters of concrete and implications for choice of testing standard. Cement and Concrete Research 2002; 32: 529-556.

[3] Bazant ZP, Yu Q, Zi G. Choice of standard fracture test for concrete and its statistical evaluation. International Journal of Fracture 2002; 118: 303-337.

[4] Bischoff PH, Perry SH. Impact behavior of plain concrete loaded in uniaxial compression. Journal of Engineering Mechanics 1995; 121(6): 685-693.

[5] Chopra AK. Dynamics of structures: Theory and applications to earthquake engineering. Prentice-Hall: Upper Saddle River, N.J, 2001.

[6] Davenne L, Ragueneau F, Mazars J, Ibrahimbegovic A. Efficient approaches to finite element analysis in earthquake engineering. Computers and Structures 2003; 81: 1223-1239.

[7] Dilger WH, Koch R, Kowalczyk R. Ductility of plain and confined concrete under different strain rates. ACI Journal 1984; 81(11): 73-81.

[8] Domínguez N, Fernández MA. Enhanced solid element for modelling of reinforced concrete structures with bond-slip. Computers and Concrete 2010; (in press).

[9] Dujc J, Brank B, Ibrahimbegovic A, Brancherie D. An embedded crack model for failure analysis of concrete solids. Computers and Concrete 2010; (in press).

[10] Garikipati K, Hughes TJR. A study of strain localization in a multiple scale framework - the one-dimensional problem. Computational Methods in Applied Mechanics and Engineering 1998; 159: 193-222. 
[11] Halphen B, Nguyen QS. On generalized standard materials (in French). Journal de Mécanique 1975; 14: 39-63.

[12] Hautefeuille M, Melnyk S, Colliat J-B. Failure model for heterogeneous structures using structured meshes and accounting for probability aspects. Engineering Computations 2009; 26(1/2): 166-184.

[13] Hillerborg A, Modéer M, Petersson PE. Analysis of crack formation and crack growth in concrete by means of fracture mechanics and finite elements. Cement and Concrete Research 1976; 6: 773-782.

[14] Hill R. The Mathematical Theory of Plasticity. Clarenden Press: Oxford, 1950.

[15] Ibrahimbegovic A. Nonlinear solids mechanics - Theoretical formulations and finite element solution methods. Springer: Berlin, 2009.

[16] Ibrahimbegovic A, Brancherie D. Combined hardening and softening constitutive model of plasticity: precursor to shear slip line failure. Computational Mechanics 2003; 31: 88-100.

[17] Ibrahimbegovic A, Boulkertous A, Davenne L, Muhasilovic M, Pokrklic A. On modeling of fire resistance tests on concrete and reinforced concrete structures. Computers and Concrete 2010; (in press).

[18] Ibrahimbegovic A, Jehel P, Davenne L. Coupled plasticity-damage model and direct stress interpolation. Computational Mechanics 2008; 42: 1-11.

[19] Kucerova A, Brancherie D, Ibrahimbegovic A, Zeman J, Bittnar Z. Novel anisotropic continuum-discrete damage model capable of representing localized failure of massive structures Part II: identification from tests under heterogeneous stress field. Engineering Computations 2009; 26(1/2): 128144 .

[20] Lu Y, Xu K. Modelling of dynamic behaviour of concrete materials under blast loading. International Journal of Solids and Structures 2004; 41: 131143.

[21] Lubliner J. Plasticity Theory. Macmillan: New York, 1990.

[22] Markovic D, Ibrahimbegovic A. Complementary energy based FE modelling of coupled elasto-plastic and damage behavior for continuum microstructure computations. Computational Methods in Applied Mechanics and Engineering 2006; 195: 5077-5093.

[23] Martinelli P, Filippou F. Simulation of the shaking table test of a sevenstory shear wall building. Earthquake Engineering and Structural Dynamics 2009; 38: 587-607.

[24] Maugin GA. The thermodynamics of nonlinear irreversible behaviors an introduction. River Edge, NJ World Scientific: Singapore, 1999.

[25] Mousseau S, Paultre P, Mazars J. Seismic performance of a full-scale, reinforced high-performance concrete building - Part II: analytical study. Canadian Journal of Civil Enginnering Engineering 2008; 35: 849-862. 
[26] Oliver J, Huespe AE. Theoretical and computational issues in modelling material failure in strong discontinuity scenarios. Computer Methods in Applied Mechanics and Engineering 2004; 193: 2987-3014.

[27] Panoskaltsis VP, Papoulia KD, Bahuguna S, Korovajchuk I. The generalized Kuhn model of linear viscoelasticity. Mechanical Time-Dependent Materials 2007; 11: 217-230.

[28] Pedersen RR, Simone A, Sluys LJ. An analysis of dynamic fracture in concrete with a continuum visco-elastic visco-plastic damage model. Engineering Fracture Mechanics 2008; 75: 3782-3805.

[29] Perzyna P. Fundamental problems in viscoplasticity. Advances in applied mechanics; 9: 243-377. Academic Press: New York, 1966.

[30] Pham BH, Davenne L, Brancherie D, Ibrahimbegovic A. Stress resultant model for ultimate load design of reinforced concrete frames - Combined axial force and bending moment. Computers and Concrete 2010; (in press).

[31] Ramtani S. Contribution to the modeling of the multi-axial behavior of damaged concrete with description of the unilateral characteristics. $\mathrm{PhD}$ thesis (in French): Paris 6 University, 1990.

[32] Simo JC, Kennedy JG, Taylor RL. Complementary mixed finite element formulations for elastoplasticity. Computer Methods in Applied Mechanics and Engineering 1989; 74: 177-206.

[33] Simo JC, Oliver J, Armero F. An analysis of strong discontinuity induced by strain softening solutions in rate-independent solids. Journal of Computational Mechanics 1993; 12: 277-296.

[34] Strang G. Introduction to Applied Mathematics. Cambridge Press: Wellesley, MA, 1986.

[35] Taylor RL (2005) FEAP: A Finite Element Analysis Program, User manual and Programmer manual, version 7.4. Department of Civil and Environmental Engineering, University of California, Berkeley, California.

[36] Wang J. Intrinsic damping: modeling techniques for engineering systems. ASCE Journal of Structural Engineering 2009; 135(3): 282-291.

[37] Weerheijm J, Van Doormaal JCAM. Tensile failure of concrete at high loading rates: new test data on strength and fracture energy from instrumented spalling tests. International Journal of Impact Engineering 2006; 34(3): 609-626. 